\title{
The minimum circuity frontier and the journey to work
}

\author{
David Levinson ${ }^{\mathrm{a}, *}$, Ahmed El-Geneidy ${ }^{\mathrm{b}, 1}$ \\ a University of Minnesota, Department of Civil Engineering, 500 Pillsbury Drive SE, Minneapolis, MN 55455 USA \\ b School of Urban Planning, McGill University, Suite 401, Macdonald-Harrington Building, 815 Sherbrooke Street West, Montreal, Quebec, Canada H3A 2K6
}

\section{A R T I C L E I N F O}

\section{Article history:}

Received 13 May 2009

Received in revised form 13 July 2009

Accepted 13 July 2009

Available online 21 July 2009

\section{Keywords:}

Network structure

Travel behavior

Transport geography

Commuting

Circuity

Directness

\begin{abstract}
A B S T R A C T
People travel between places of residence and work destinations via transportation networks. The relation between selection of home and work locations has been heavily debated in the transportation planning literature. In this paper we use circuity, the ratio of network to Euclidean distance, to better understand the choice of residential location relative to work. This is done using two methods of defining origins and destinations in twenty metropolitan regions in the United States, with more detailed analysis of Minneapolis-St. Paul, Minnesota and Portland, Oregon. The first method of selection is based on actual choice of residence and work locations. The second is based on a randomly selected dataset of origins and destinations in the same regions, followed by a comparison between the two methods for these regions. The study shows circuity measured through randomly selected origins and destinations differs from circuity measured from actual origins and destinations. Workers tend to reside in areas such that the journey to work circuity is lower than random, applying intelligence to their location decisions. Consistent with traditional urban economic theory, this suggests locators wish to locate on the frontier with the largest residential lot at the shortest commute time, but in contrast with the classic model which simplifies transportation networks to be uniform, we cannot assume that all possible home-work pairs are on the frontier. This finding, developed from microscopic data not previously used for this question, reveals an important issue related to residence choice and location theory and how resident workers tend to locate with respect to network configuration in an urban context.
\end{abstract}

(C) 2009 Elsevier B.V. All rights reserved.

\section{Introduction}

"Man walks in a straight line because he has a goal and knows where he is going; he made up his mind to reach some particular place and he goes straight to it" (Le Corbusier, 1929).

The presence of a transportation system (including networks and modes) dissuades people from traveling to their destinations in a straight line by providing the opportunity to move faster if more circuitously; while buildings, and other infrastructure (including transportation networks when they act as barriers), and features of nature such as rivers and mountains may constrain the direction of movement.

Distances in transportation research can be measured using geographic information systems (GIS) in three forms: Euclidean distance, network distance, and Manhattan distance. Manhattan distance is not commonly used in transportation research since it is generally meaningful only on a grid system, which holds strictly in few urban contexts. Euclidean distance is the airline distance measured between origins and destinations "as the crow flies", while the

\footnotetext{
* Corresponding author. Tel.: +16126256354.

E-mail addresses: dlevinson@umn.edu (D. Levinson), ahmed.elgeneidy@mcgill.ca (A. El-Geneidy).

URL's: http://nexus.umn.edu (D. Levinson), http://tram.mcgill.ca (A. El-Geneidy).

1 Tel.: +1 5143988741 .
}

network distance, which is a more realistic representation of movements between origins and destinations, is the distance between origins and destinations measured along a transportation network, usually using the shortest path (Miller and Shaw, 2001), these are shown in Fig. 1.

Circuity, the ratio of network to Euclidean distance, has been examined by a number of researchers in a variety of contexts. Love and Morris (1979) estimate road distance between two points using analytic models primarily for facilities location problems. Newell (1980) indicated that network distance measured for a randomly selected set of points in an urban environment is about 1.2 times the Euclidean distance. Other research (O'Sullivan and Morrall, 1996) finds circuity factors of 1.21 to 1.23 at various transit station catchment areas. The measure has also been used at the national level (Ballou et al., 2002), and for pedestrian and bicycle travel (dubbed pedestrian route directness) (Dill, 2003), with much higher values than observed for automobile travel. The measure has also been considered by Wolf et al. (2004) using GPS traces of actual travelers route selections, finding that many actual routes experience much higher circuity than might be expected. Samaniego and Moses (2008) find that road networks are built as if traffic were completely decentralized, while travel itself remains mixed between centrality (all destinations in a central business district) and decentralization (trips go to the nearest destination), perhaps explaining some of the observed circuity. Selection of any random pair of points 
a

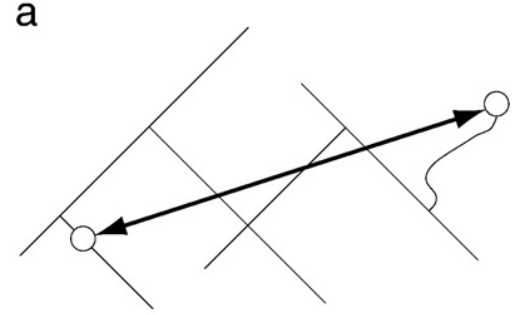

b

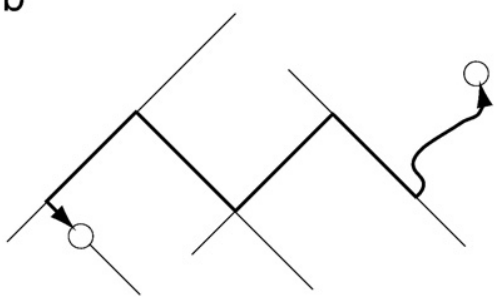

Fig. 1. Difference between Euclidean and network distances.

in an urban environment and measuring circuity may lead to a different answer than the circuity experienced from an actual selection of an origin and destination by locator-travelers.

A question that arises is whether the differences between Euclidean distance and network distance are small and constant. This research tests that proposition, positing that this assumption only holds when variation in the network is minor and when self-selection is not present. The issue of self-selection has largely been neglected in analysis of network circuity. While it is commonly understood that residents choose homes considering attributes of accessibility to work, shopping, schools, amenities, quality of neighborhood life, availability of public service, quality of the house (number of bedrooms, bathrooms, etc.) and costs of living (McFadden et al., 1978), the implications of this for measurement of circuity have not previously been noted in the literature. An analysis of circuity bears on the question of home-work location.

The relationship between home-work locations is complicated and has been heavily debated in the literature. The standard model of urban economics states that choice of residential location is based on tradeoff between commuting cost and land cost (Mills, 1972). Embedded within the model are behavioral assumptions that have been challenged. Residential location preferences also depend on access to destinations such as schools, shopping, and other amenities. Small and Song (1992) and Giuliano and Small (1993) concluded that the behavioral assumption of cost minimization for just the journey to work in the standard model is inadequate when explaining the relation between job and housing location, while Giuliano (1991) suggests that home selection may have little to do with job access considerations. On the other hand Wachs et al. (1993) found that several factors affect home selection locations including the homework separation. Clark et al. (2003) indicate that, even in two-worker households, workers try to minimize their commuting distance to work. Recently Kim (2008) found that when people change their work locations, they prefer selecting jobs with similar commuting distances or time compared to their previous job. In addition telecommuting may affect choice of residential location (Tayyaran et al., 2003). van Ommeren (2004) shows that there are notable frictions in the choice of job and housing due to search and transaction costs, so people do not live and work in optimal locations. The extent to which that plays out in network circuity is unclear, but suggests that observed circuity is higher than it would be if locations were optimal. Further, Redmond and Mokhtarian (2001) observe that people have a positive optimal commute distance, suggesting people with a short Euclidean distance may not object to a greater circuity. Overall, the extent to which work location affects the selection of home location (and vice versa) remains unsettled. Even if location depends on multiple factors, work remains significant for many locators. Our research complements much of the previous debate about wasteful commuting by examining network structure. However it differs in that the wasteful commuting argument posits what would happen if people located to minimize commute distance (or time), while we examine how people can maximize living space while minimizing time by orienting their commute along a network in a particular way.

The paper next details the research design. Then it includes a brief description of the data sets used in the analysis. Circuity is measured for Minneapolis-St. Paul (Twin Cities), Minnesota and Portland, Oregon. It next constructs a model to predict the network circuity as a function of network structure and transportation geography. The circuity analysis is applied to twenty US metropolitan areas for comparison. Finally conclusions are drawn.

\section{Research design}

In the standard monocentric urban economic model, house location relative to work is identical to house location relative to the center of the city, and thus how much land costs. The cost of land tends to decrease with the increase in Euclidean distance from the center, while keeping other factors affecting land value constant. Network distance, on the other hand, is an indicator of how much travel actually takes place (and is more closely related to travel time), which has implications for congestion, pollution, and travel behavior and activity patterns. The commute time tends to increase with the increase in the network distance, while keeping all other factors affecting travel time constant. This relation is described in Fig. 2.

Cities of course are not monocentric (Cho et al., 2008), yet if Euclidean distance between home and work is still correlated with land and housing costs, individuals who choose greater Euclidean distances should be able to improve the quality and size of their home, ceteris paribus. Individuals who minimize home-work circuity lie on the frontier with the maximum house and lot at the minimum travel time (as the network distance is closer to a straight line). We posit that individuals would like to have the most space available at the least travel and monetary cost. This implies that, for a given indifference curve, home-work pairs will be chosen where the network circuity is at a minimum. We expect to find that circuity is lower for actual home-work pairs than for the random set of origin-destination pairs that have been used in previous research (Newell, 1980), as people can select how to arrange their activities on the network.

To test this hypothesis we compare circuity using several sets of origins and destinations. The first is a set of origins and destinations defined based on actual home-work pairs (Case 1). The second dataset uses the same origins and destinations, but randomizes the matching of origins and destinations, and so is most analogous to previous analyses of network circuity. This is done in a stepwise method to

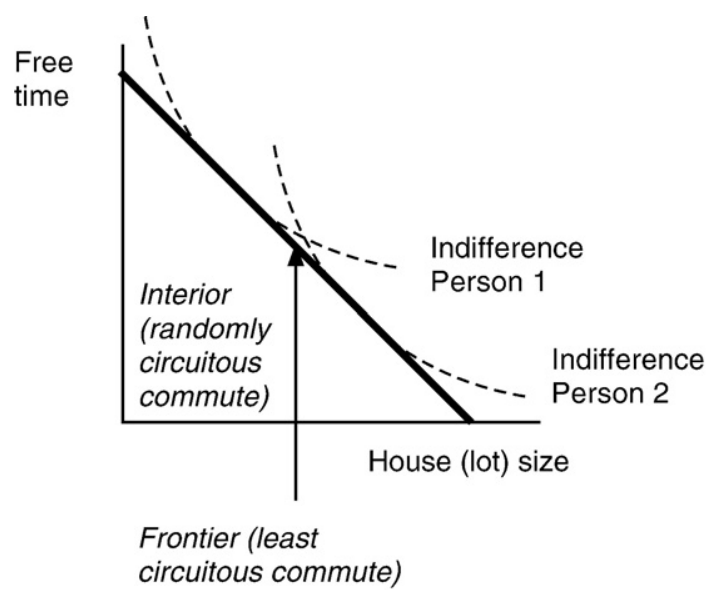

Fig. 2. Relationship between home-work location and travel time and house size. 
Table 1

Summary of cases.

\begin{tabular}{lll}
\hline & Euclidean distances & \\
\cline { 2 - 3 } Network distances & Fixed to Observation & Random \\
\hline Fixed to observation & Case 1 (Observed) & Case 4 \\
Random & Case 3 & Case 2 (Literature) \\
\hline
\end{tabular}

capture the effects of various distances between random origins and destinations (Case 2). The third dataset uses the same origins and destinations, but randomizes the matching while trying to ensure the same statistical distribution of network distance, allowing the Euclidean distance to vary (Case 3 ). The fourth uses the same origins and destinations but randomizes the matching, in this case retaining the same statistical distribution of Euclidean distance but allowing the network distances to vary (Case 4). These are summarized in Table 1.

We expect that circuity in Case 1 is the lowest of the four cases, as that case represents intention on the part of travelers. In addition the research will predict the network distance of OD pairs as a function of Euclidean distance, while controlling for location of origin and destination and interaction of the two, and type of network present. To ensure the robustness of our findings, the same methods will be tested in twenty-two different metropolitan regions in the United States.

\section{Data}

The Longitudinal Employer-Household Dynamics dataset (LEHD) used here is a comprehensive dataset that includes peoples place of residence identified at the Census Block level of analysis and their employment location identified at the same level. The LEHD data set for the Twin Cities region collected in 2002 contains 1,422,980 observations aggregated at the census block level of analysis. This home-work pair matrix contains records with multiple workers sharing both origin and destination block. Pairs with more than one trip are converted to individual trip records to generate a new data set with 2,377,157 actual home-work pairs. In order to decrease the complexity of calculations a random sample of resident workers is selected (5000 observations) from the LEHD dataset. From the 2,377,157 a sample of 5000 home-work pairs is selected to be used in the calculation of case 1. Both Euclidean and network distances are calculated for the 5000 home-work pairs.

To prepare data for cases 2, 3 and 4, two new samples are generated. The first includes 200 randomly distributed points in the region, while the second includes 1000 randomly distributed points, this is equivalent to the

Table 2

Network Distance $=f($ Euclidean Distance $)$ models for the Twin Cities region.

\begin{tabular}{lllllll}
\hline Case & Description & $\begin{array}{l}\text { Circuity } \\
\text { coefficient }\end{array}$ & $R^{2}$ & $\begin{array}{l}\text { Average network } \\
\text { distance }\end{array}$ & $\begin{array}{l}\text { Average Euclidean } \\
\text { distance }\end{array}$ \\
\cline { 5 - 5 } & & & & $(\mathrm{m})$ & \\
\hline 1 & & Home-work & 1.18 & 0.99 & 17,845 & 14,746 \\
3 & Euclidean distance & 1.22 & 0.99 & 18,134 & 14,357 \\
& matched & & & & \\
4 & Network distance & 1.25 & 0.98 & 19,473 & 14,987 \\
& matched & & & & \\
2 & Euclidean distance & & & & \\
$2-1$ & $\leq 5 \mathrm{~km}$ & 1.58 & 0.85 & 5250 & 3295 \\
$2-2$ & $>5 \mathrm{~km}$ and $\leq 10 \mathrm{~km}$ & 1.42 & 0.94 & 11,021 & 7731 \\
$2-3$ & $>10 \mathrm{~km}$ and $\leq 15 \mathrm{~km}$ & 1.34 & 0.97 & 16,986 & 12,639 \\
$2-4$ & $>15 \mathrm{~km}$ and $\leq 20 \mathrm{~km}$ & 1.30 & 0.98 & 22,845 & 17,549 \\
$2-5$ & $>20 \mathrm{~km}$ and $\leq 25 \mathrm{~km}$ & 1.27 & 0.98 & 28,660 & 22,558 \\
$2-6$ & $>25 \mathrm{~km}$ and $\leq 30 \mathrm{~km}$ & 1.25 & 0.99 & 34,376 & 27,539 \\
$2-7$ & $>30 \mathrm{~km}$ and $\leq 35 \mathrm{~km}$ & 1.23 & 0.99 & 40,072 & 32,536 \\
$2-8$ & $>35 \mathrm{~km}$ and $\leq 40 \mathrm{~km}$ & 1.22 & 0.99 & 45,762 & 37,554 \\
$2-9$ & $>40 \mathrm{~km}$ and $\leq 45 \mathrm{~km}$ & 1.21 & 0.99 & 51,267 & 42,519 \\
$2-10$ & $>45 \mathrm{~km}$ and $\leq 50 \mathrm{~km}$ & 1.2 & 0.99 & 56,745 & 47,457 \\
\hline
\end{tabular}

$N=5000$.

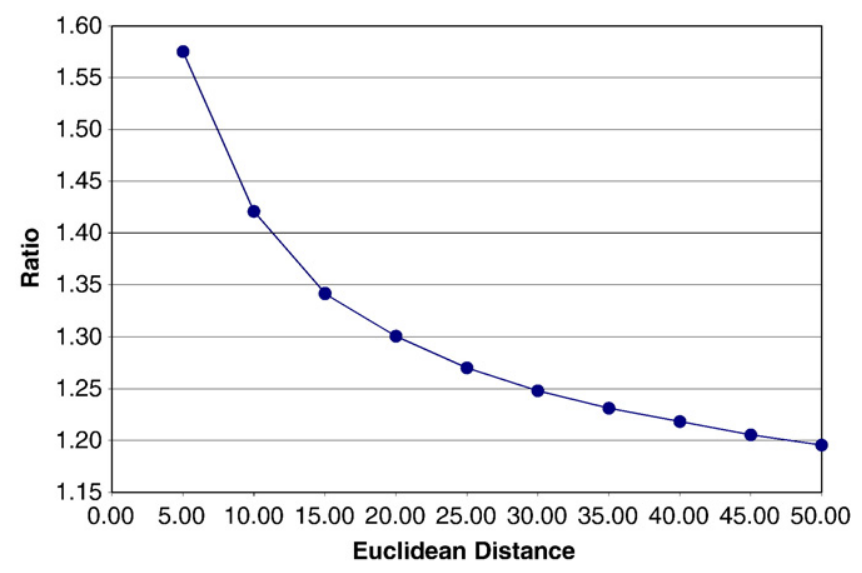

Fig. 3. Circuity (the ratio between network and Euclidean distance) using random sample stratified by Euclidean distance $(\mathrm{km})$.

ratio of work to home locations in the region. Both Euclidean and network distances are calculated for the entire random matrix, generating 200,000 OD pairs. In order to generate data for cases 3 and 4, Euclidean and network distances are rounded to the nearest $10 \mathrm{~m}$ in both matrices (random and home-work). From the random 200,000 OD pair matrix a sample of 5000 observations is selected that have the same distribution of Euclidean distances as the observed sample. This sample is used to generate the data needed for case 3 . Similarly a sample of 5000 OD pairs is selected from the random 200,000 OD pair matrix, while fixing the distribution of network distances to generate data for case 4 . Meanwhile for case 2 a random sample of 5000 observations is selected from the 200,000 OD pair matrix.

Since Census Block sizes are fairly small (100 by $100 \mathrm{~m}$ or even smaller), Euclidean and network distance are calculated from the centroid of each census block. An implicit assumption is that travel takes place on the shortest distance path along the road network, though we do not know the actual mode or path used for travel in the LEHD database. Accordingly we use the shortest network distance between two points as a proxy for the actual network distance. We recognize it is likely that individuals do not actually take the shortest distance path (Wolf et al., 2004) due to congestion, or even the shortest travel time path, for a variety of reasons (information, travel time reliability, cost, preferences for other route attributes). This assumption nevertheless provides a useful starting point for comparison of many different commutes given the available data.

\section{Measuring circuity}

Using network distance as the dependent variable and Euclidean distance as the independent variable, while assigning the value of zero to the intercept, regression models are estimated for each case. Table 2 includes the output of the regression analyses showing the network circuity and the average network and Euclidean distances. All models had a sample

Table 3

Network Distance $=f$ (Euclidean Distance) Models for Portland Oregon.

\begin{tabular}{|c|c|c|c|c|c|}
\hline \multirow[t]{2}{*}{ Case } & \multirow[t]{2}{*}{ Description } & \multirow[t]{2}{*}{$\begin{array}{l}\text { Circuity } \\
\text { coefficient }\end{array}$} & \multirow[t]{2}{*}{$R^{2}$} & \multirow{2}{*}{$\begin{array}{l}\text { Average network } \\
\text { distance } \\
(\mathrm{m})\end{array}$} & \multirow{2}{*}{$\begin{array}{l}\text { Average euclidean } \\
\text { distance } \\
(\mathrm{m})\end{array}$} \\
\hline & & & & & \\
\hline 1 & Home-work & 1.19 & 0.99 & 14,472 & 11,848 \\
\hline 2 & Random & 1.22 & 0.99 & 35,648 & 29,067 \\
\hline 3 & $\begin{array}{l}\text { Euclidean distance } \\
\text { matched }\end{array}$ & 1.23 & 0.98 & 15,084 & 12,090 \\
\hline 4 & $\begin{array}{l}\text { Network distance } \\
\text { matched }\end{array}$ & 1.21 & 0.99 & 14,908 & 12,143 \\
\hline
\end{tabular}

$N=5000$. 


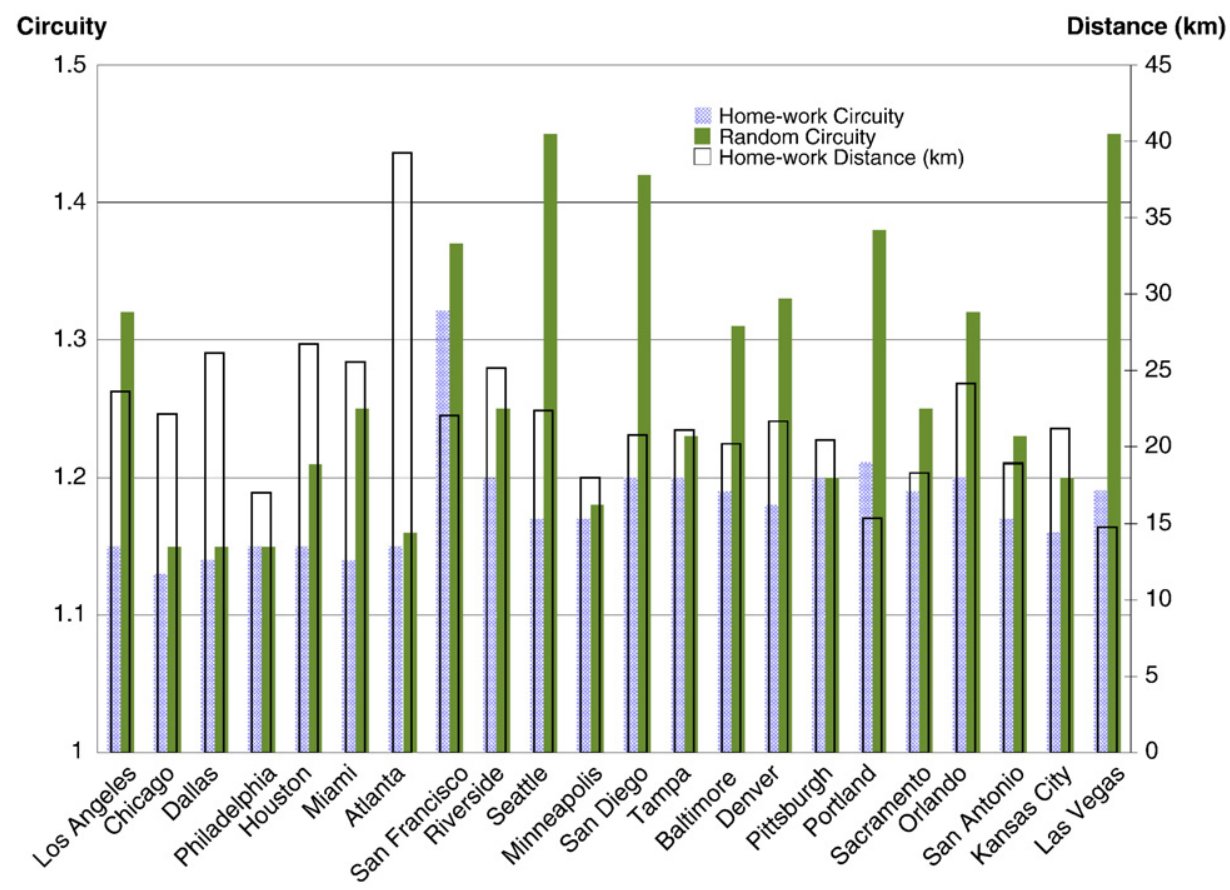

Fig. 4. Circuity in twenty-two US cities.

size of 5000 observations. Case 2 is divided into ten subsamples that will be selected randomly from the 200,000 sample. Each sample includes 5000 observations while limiting the Euclidean distance to be in a certain range. For example selecting a sample of 5000 observations where the Euclidean distance is greater than $5 \mathrm{~km}$ and less than $10 \mathrm{~km}$.

The analysis shows that differences between Euclidean and network distances measured through randomly selected origins and destinations tend to differ from distances measured based on home-work location. The pairs derived based on the home-work relationship has a lower average circuity (1.18) compared to all the other randomly selected points ( 1.22 for case $3,1.25$ for case 4 ). This observation holds even when matching the Euclidean or network distances. In other words, workerlocators tend to choose home-work pairs where the circuity is lower, applying intelligence to their location decisions. This finding reveals an important issue related to residential location choice theory in an urban context: the efficiency of the network cannot be assessed independently of how travelers use it. A t-test demonstrates that the home-work relation tends to be statistically different from all the other randomly observed measures of circuity at the $99 \%$ confidence interval. Observing the ten samples of case 2, the circuity decreases among the randomly selected O-D pairs with the increase of both Euclidean and Network distances. This relationship is illustrated in Fig. 3.

As Redmond and Mokhtarian (2001) show a positive utility to commutes, it may be that people with shorter commutes prefer them to be more circuitous to add time, and so people who locate near (in a Euclidean sense) to their workplace might select for networks with more circuitous paths, though that hypothesis cannot be fully examined here. It is more likely the increased circuity associated with shorter trips is largely a function of network structure, as it appears in both the actual and random set of paths.

Next we compare the circuity in the Twin Cities with metropolitan Portland, Oregon again using 2002 LEHD data. The reason for selecting Portland is due to the availability in LEHD data for this region and the similarities between these cities. (The cities are mid-size (1.6 million workers in Minneapolis, 1.1 million in Portland as of 2000), both on rivers (Mississippi and Willamette respectively), the metro area drive alone mode-share in Minneapolis is 78.3\%, in Portland it is 73.1\%) and have an original economic base in resource extraction which has moved to higher technology and service industries.
A random sample of 5000 home to work observations was selected from the Portland LEHD data to conduct this comparative analysis. Similar to the Twin Cities analysis a set of randomly generated points is used as origins and destinations for cases 2 through 4 . Table 3 shows the circuity analysis for the Portland region using a similar methodology to the one used in the Twin Cities region, but limiting case 2 to one observation rather than breaking it to ranges. The first observation is that differences exist in average home to work travel length between the two regions. Workers in the Twin Cities travel approximately $3 \mathrm{~km}$ more than workers residing in the Portland region. It is clear that although differences exists between the two regions in term of planning policies (Portland has a famous urban growth boundary limiting its growth, which encourage increase in densities) the circuity is almost the same (1.19 for case 1 in Portland compared to 1.18 in the Twin Cities). Yet differences exist when comparing Cases 2, 3 and 4 from the Portland region to the Twin Cities region. These differences are due to the differences in the network structure, which reflects differences in planning policies and market conditions that these cities were subject to over time.

The analysis was replicated for an additional 20 US metropolitan areas (denoted by their primary city, ranked from largest on the left to smallest on the right) with available LEHD data (for comparability using 2003 LEHD data for all cities) ${ }^{2}$, corroborating the general findings above, as shown in Fig. 4. In all cities, actual circuity was lower than random points would provide, in some cases noticeably so. Circuity was highest in the San Francisco region, which may be driven by the unusual geography of the metropolitan area (land surrounding a bay) and a limited number of bay crossings. A difference of means $t$-test indicates the two series (Case 1: home-work circuity (mean 1.18) and Case 2: random circuity (mean 1.27) are significantly different, with a $t$-statistic of 5.0. (Similar results would be seen for Case 3 and Case 4 in comparison with Case 1).

As can be observed in the figure, the smaller cities on the right have in general a slightly higher average circuity than the larger cities, they

\footnotetext{
2 Data was not available in whole or in part for New York, Washington, Boston, Cincinnati, and Cleveland regions, and was problematic in Detroit and St. Louis, so those cities were excluded from the analysis. Most of those metropolitan areas straddle more thanone state (or in the case of Detroit, province), explaining some of the data issues, while only two of the 22 metropolitan areas with good data are multi-state.
} 
also have a shorter average network distance (since long trips in smaller areas are less likely than long trips in larger areas, as one more quickly reaches the edge of the developed region). This is shown with the home to work network distance shown on the second $y$-axis, which tends to be highest for the larger cities (Atlanta is the highest, Portland and Las Vegas are the lowest).

While the models in this section show the general trends in the differences in circuity between randomly selected sets of origins and destinations and home-work location, this method of analysis does not account for the network structure. A model is introduced in the following section to help in understanding the differences between circuity of home-work location and circuity for randomly selected set of points, while controlling for the variation in the network structure. The sample used in this analysis includes 5000 observed home-work pairs in addition to 5000 randomly selected origin and destination pairs.

\section{Explaining circuity}

In order to account for the variation in network structure in the Twin Cities, the region is divided into four different rings. Different street network topologies characterize each ring. The urban ring, which includes the cities of Minneapolis and Saint Paul (ring 1) has the most grid-like network. The second near-urban area transitions from a grid-like network to the tree-like system (with unconnected

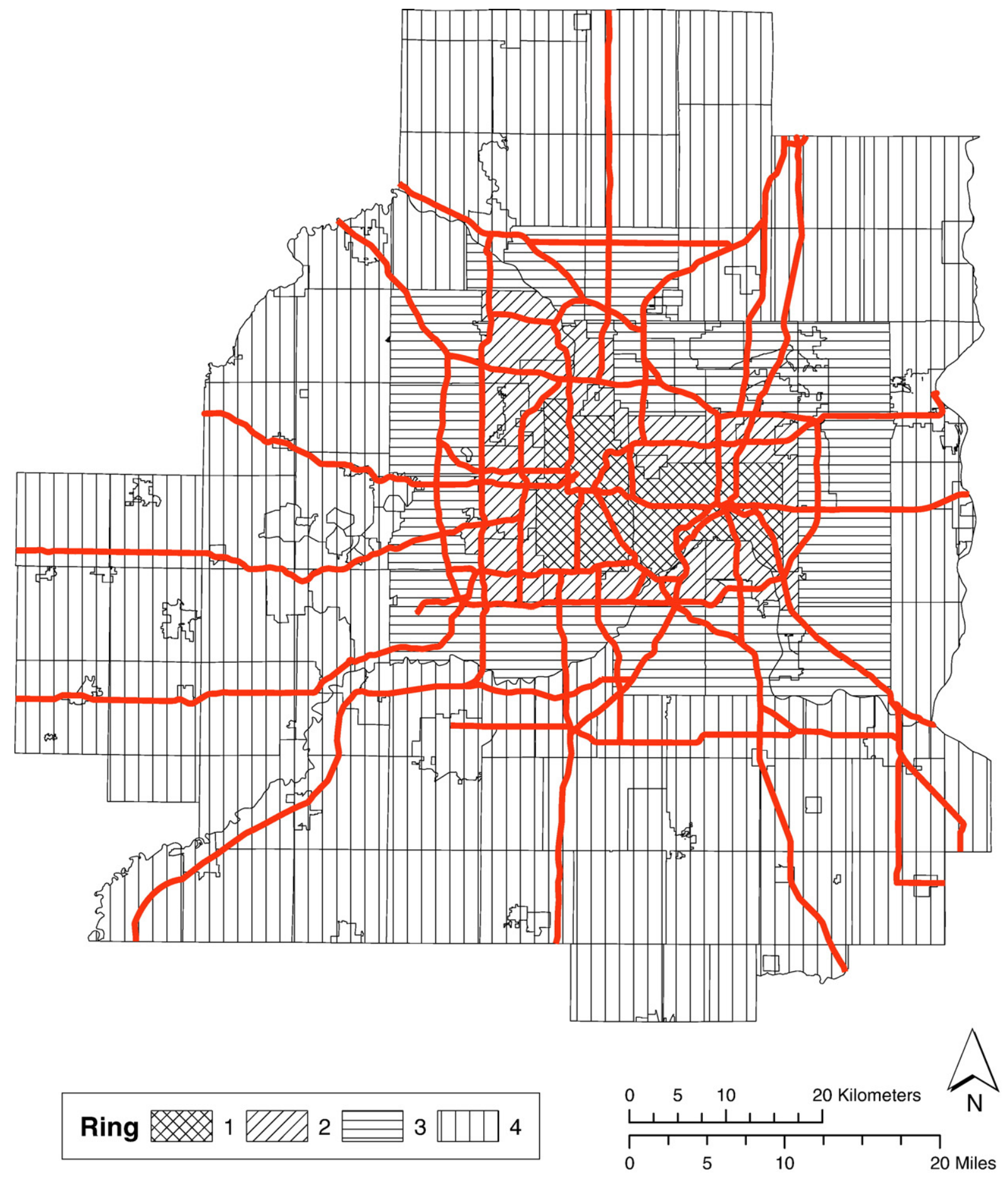

Fig. 5. Twin Cities region divided into rings. 
Table 4

Circuity $=f$ (Network Attributes) model for the Twin Cities region.

\begin{tabular}{lcc}
\hline Independent variable & Coefficient & $t$-Stat \\
\hline Intercept & 1.4273 & 161.64 \\
Number of street-street nodes & 0.0002 & 9.5 \\
Number of street-freeway nodes & -0.0001 & -1.15 \\
Number of freeway-freeway nodes & 0.0006 & 8.91 \\
Freeway length (km) & -0.0013 & -10.34 \\
Street length (km) & -0.001 & -11.45 \\
Network distance (km) & 0.0061 & 13.51 \\
Dummy if buffer intersects ring 1 & -0.0096 & -1.22 \\
Dummy if buffer intersects ring 2 & 0.0136 & 1.74 \\
Dummy if buffer intersects ring 3 & 0.0097 & 1.49 \\
Dummy if buffer intersects ring 4 & -0.0508 & -6.78 \\
Home-work dummy & -0.0568 & -8.99 \\
\hline$R^{2}=0.11, N=10,000$ & &
\end{tabular}

$R^{2}=0.11, N=10,000$.

branches emanating from the main network $)^{3}$ (ring 2). The suburban ring has network topologies which are more tree-like (ring 3). Finally the rural areas which are beyond the major freeway system have a grid-like network, but the grid is at a much larger scale than in the center city (ring 4). Fig. 5 maps the rings.

A 2 kilometer buffer is generated around the line representing the Euclidean distance. This buffer is used to calculate the characteristics of the network in the area between origins and destinations. Characteristics include the number of nodes generated due to intersections between streets, streets and freeways, and/or freeways with freeways. The length of freeways and streets in the buffer are also included in the characteristics section. The shortest network distance between each origin and destination is included to account for the length of the trip. A set of dummy variables represents which ring in the region the line representing the Euclidean distance intersects. Many variables affect circuity: one-way streets would increase local circuity, though in the Twin Cities these are found only in the central cities and so not tested directly; other attributes of network structure can be posited, but the number of nodes and length of links gets at many of these (e.g. high density grids have more nodes per unit length, tree-like networks have fewer). The home-work dummy is added to identify actual OD pairs distinct from random OD pairs. Table 4 contains the output of the model.

From Table 4 it is clear that if the line measuring the Euclidean distance crosses rings 1 or 4 a negative effect is present, while crossing rings 2 or 3 a positive effect is present. (Some buffers intersect multiple rings, allowing us to avoid correlation problems associated with the dummy variable trap.) This observation indicates the presence of a unique network structure in each ring. Ring 1, which represents the core cities of Minneapolis and Saint Paul, has a well connected grid system, as does the rural ring 4 (though at a lower density). Rings 2 and 3 have more tree-like, and less direct, suburban road networks. The circuity effect on a person residing in ring 1 and working in ring 4 can be obtained from combining the effects of passing by all four rings (the four coefficients together).

Surprisingly, the number of street-street nodes and freewayfreeway nodes have a statistically significant and positive effect on circuity. Both variables are indicators of the density of the network. Where the network is denser, but gridded, the circuity may be higher than a less dense, but non-90 degree oriented network, e.g. where more radial routes travel on the hypotenuse of the triangle rather than following right angles. In addition freeways often have features that add to circuity (e.g. Cloverleafs) but lower travel time. Meanwhile the number of nodes representing intersections between the freeway system and the street system has a statistically insignificant (though negative) effect on circuity.

\footnotetext{
3 Tree-like networks may be radial if the trunk of the tree is the central city, however, radial networks may be connected by rings, so are not necessarily tree-like.
}

Both freeway length and street length have a statistically significant negative effect on circuity, so the more roads, the more direct the path possible, which is expected. The actual network distance, which is included as a control variable to represent scale, shows a statistically significant positive effect on circuity, which is an expected effect. The home-work dummy variable did show a statistically significant negative effect on circuity, supporting the argument that travelers apply intelligence compared to random points. This indicates that circuity along a pair that is obtained based on home-work relation is lower than circuity obtained from random cases by 0.057 .

\section{Conclusions}

In this paper we use network circuity, the ratio of network to Euclidean distance, to better understand the choice of home-work pairs. This is done using two methods of defining origins and destinations: the actual choice of residence and work locations, and a randomly selected dataset of origins and destinations. The findings of the study show that circuity measured through randomly selected origins and destinations exceeds circuity measured from actual home-work pairs. Workers tend to choose commutes with lower circuity, applying intelligence to their home location decisions compared to their work. We posit this is because locators wish to achieve the largest residential lot for the shortest commute time, all else equal.

The results indicate that people in the Twin Cities region are selecting network circuity ratios that are 0.057 smaller than random, while controlling all other factors affecting the selection of home locations. While that number may not sound large, it represents a $25 \%$ improvement in home-work circuity compared to the random sample (since the best possible ratio would be 1.0). Similarly in Portland the observed home-work circuity 1.19 represents a $17 \%$ improvement over the random 1.22 circuity (case 2 ). The result is corroborated in twenty other US metropolitan areas.

Given all of the other constraints individuals face when finding housing and jobs in a multi-worker context and on inefficient networks, we conclude that maximizing land while minimizing commute remains an important factor in urban location decisions. This agrees with some previous studies (Clark et al., 2003) and is in contrast with other research (Giuliano, 1991; Giuliano and Small, 1993). People account for network topology when making location-choice decisions, indicating simple distance metrics should not be used in travel behavior and planning models. An important corollary of these findings is that the efficiency of the network cannot be assessed independently of how travelers use it.

\section{Acknowledgment}

The authors would like to thank Lisa Kastelberger, McGill University for assistance with the literature review. Jessica Horning, now at Cambridge Systematics, assisted with the analysis of Portland while working at the University of Minnesota. The analysis for US cities aside from Minneapolis-St. Paul and Portland was computed by Farhana Yasmin, a PhD student at McGill School of Urban Planning. We would also like to thank several anonymous reviewers for their helpful comments.

\section{References}

Ballou, R., Rahardja, H., Sakai, N., 2002. Selected country circuity factors for road travel distance estimation. Transp. Res. Part A 36 (9), 843-848.

Cho, E., Rodriguez, D., Song, Y., 2008. The role of employment subcenters in residential location decisions. J. Transp. Land Use 1 (2), 121-151.

Clark, W., Huang, Y., Withers, S., 2003. Does commuting distance matter? Commuting tolerance and residential change. Reg. Sci. Urban Econ. 33 (2), 199-221.

Dill, J., 2003. Measuring network connectivity for bicycling and walking. Unpublished paper presented at Joint Congress of ACSP-AESOP, Leuven, Belgium, July. Available online at: http://www.enhancements.org/download/trb/trb2004/TRB2004001550.pdf (access date July 26, 2009).

Giuliano, G., 1991. Is jobs-housing balance a transportation issue? Transp. Res. Rec. 1305, 305-312. 
Giuliano, G., Small, K., 1993. Is the journey to work explained by urban structure? Urban Stud. 30 (9), 1485-1500.

Kim, C., 2008. Commuting time stability: a test of a co-location hypothesis. Transp. Res. Part A 42 (3), 524-544.

Le Corbusier, 1929. The city of to-morrow and its planning. Dover Publications, p. Clarke, Translator.

Love, R., Morris, J., 1979. Mathematical models of road travel distances. Manage. Sci. 130-139.

McFadden, D., et al., 1978. Modelling the choice of residential location. Spat. Interact. Theory Plan. Models 25, 75-96.

Miller, H., Shaw, S., 2001. Geographic information systems for transportation: principles and applications. Oxford University Press, USA.

Mills, E., 1972. Studies in the structure of the urban economy. The Johns Hopkins Press, Baltimore, Maryland.

Newell, G., 1980. Traffic flow on transportation networks. MIT Press, Cambridge, Mass.

O'Sullivan, S., Morrall, J., 1996. Walking distances to and from light-rail transit stations. Transp. Res. Rec. 1538, 19-26.
Redmond, L., Mokhtarian, P., 2001. The positive utility of the commute: modeling ideal commute time and relative desired commute amount. Transportation 28 (2), 179-205. Samaniego, H., Moses, M., 2008. Cities as organisms: allometric scaling of urban road networks. J. Transp. Land Use 1 (1), 21-39.

Small, K., Song, S., 1992. “Wasteful” commuting: a resolution. J. Polit. Econ. 888-898.

Tayyaran, M., Khan, A., Anderson, D., 2003. Impact of telecommuting and intelligent transportation systems on residential location choice. Transp. Plan. Technol. 26 (2), 171-193.

van Ommeren, J., 2004. On commuting. Tinbergen Magazine 6-9.

Wachs, M., Taylor, B., Levine, N., Ong, P., 1993. The changing commute: a case-study of the jobs-housing relationship over time. Urban Stud. 30 (10), 1711-1729.

Wolf, J., Schoenfelder, S., Samaga, U., Oliveira, M., Axhausen, K., 2004. Eighty weeks of global positioning system traces: approaches to enriching trip information. Transportation Research Record: J. Transp. Res. Board 1870, 46-54. 